\title{
Comparison of available measurements of the absolute air-fluorescence yield and determination of its global average value
}

\author{
J. Rosado, F. Blanco, F. Arqueros \\ Departamento de Física Atómica, Molecular y Nuclear, Facultad de Ciencias \\ Fisicas, Universidad Complutense de Madrid, E-28040 Madrid, Spain
}

\begin{abstract}
Experimental results of the absolute air-fluorescence yield are given very often in different units (photons/MeV or photons $/ \mathrm{m}$ ) and for different wavelength intervals. In this work we present a comparison of available results normalized to its value in photons/MeV for the $337 \mathrm{~nm}$ band at $1013 \mathrm{hPa}$ and $293 \mathrm{~K}$. The conversion of photons/m to photons/MeV requires an accurate determination of the energy deposited by the electrons in the field of view of the experimental set-up. We have calculated the energy deposition for each experiment by means of a detailed Monte Carlo simulation and the results have been compared with those assumed or calculated by the authors. As a result, corrections to the reported fluorescence yields are proposed. These corrections improve the compatibility between measurements in such a way that a reliable average value with uncertainty at the level of $5 \%$ is obtained.
\end{abstract}




\section{Introduction}

The air-fluorescence yield $Y$, defined as the number of photons per unit of energy deposited by the shower in the atmosphere, is a key calibration parameter which determines the energy scale of fluorescence telescopes. A number of absolute measurements of the fluorescence yield have been carried out in laboratory experiments in recent years. In these experiments a beam of charged particles crosses a collision chamber filled with air at known conditions, generating fluorescence radiation which is measured by an appropriate optical system. Many authors [1, 2, 3, 4, 5, have used electrons from a source of ${ }^{90} \mathrm{Sr}$ with energy around $1 \mathrm{MeV}$. Other absolute measurements have been performed with higher energy electrons from accelerators [4, 6]. Finally the AIRFLY collaboration has carried out accurate measurements using a $120 \mathrm{GeV}$ proton beam and a preliminary value is presented in these Proceedings [7]. In table 1] a summary of available measurements is shown.

The atmospheric fluorescence emission in the spectral range of interest, i.e., $\sim 300-400 \mathrm{~nm}$, basically comes from the Second Positive (2P) system of $\mathrm{N}_{2}$ and the First Negative $(1 \mathrm{~N})$ system of $\mathrm{N}_{2}^{+}$. The excitation cross section of the $2 \mathrm{P}$ system, dominant at atmospheric pressure, peaks at about $15 \mathrm{eV}$ decreasing strongly with an $E^{-2}$ dependence. As a consequence, high-energy electrons themselves are very inefficient for the generation of air fluorescence. In fact, fluorescence emission along the track of an energetic charged particle is mainly induced by low-energy secondary electrons ejected in successive ionization processes [8, 9, 10].

Comparison of the absolute values of the fluorescence yield is not trivial. For instance some authors measure single intense fluorescence bands while others detect the integrated fluorescence in a wide spectral range. In this work we have normalized the available measurements of the absolute fluorescence yield to their values for the $337 \mathrm{~nm}$ band using experimental relative intensities [11] in good agreement with theoretical predictions [10]. Some measurements are given in units of $\mathrm{ph} / \mathrm{m}$, and converted by the authors into $\mathrm{ph} / \mathrm{MeV}$ assuming that beam electrons deposit all their lost energy in the field of view of the collision chamber. We have calculated the energy deposition in these experiments using a simulation algorithm [10] which has provided us with the appropriate corrections factors. Other experiments have determined accurately the energy deposition as well as the geometrical factors by means of well known MC codes, e.g., GEANT4 and EGS4. In these cases our simulation predictions have been compared with those of the corresponding 
experiments. In general, a reasonable agreement has been found, and thus, the corresponding corrections factors that we propose, to be consistent with our simulations, are small.

Unlike other MC codes like GEANT or EGS, our simulation algorithm [10] has been developed to treat individual interactions of both primary and secondary electrons with the molecules of the medium. All processes giving rise to energy deposition are included. Molecular excitation for the emission of $2 \mathrm{P}$ and $1 \mathrm{~N}$ photons are treated separately in such a way that fluorescence emission can be also calculated and therefore a theoretical fluorescence yield can be obtained too. GEANT4 simulations of energy deposition in air, carried out by MACFLY [4] and AIRFLY [12] are in agreement with our calculations at the level of $2 \%$ (1\%) for electron energies in the $\mathrm{GeV}(\mathrm{MeV})$ range.

By means of this algorithm we have performed two kind of simulations, i.e., generic simulations where primary electrons are forced to interact in the center of a sphere of radius $R$ filled with air at given pressure and detailed simulations [13] including the geometry as well as other experimental features. By comparison with the detailed ones we have found that even the generic simulations provide accurate results on energy deposition as far as the radius of the sphere is a reasonable representation of the size of the experimental set-up. This behavior is expected since energy deposition is a weak function of $R[10]$.

As a result of our calculations, normalized values of the air-fluorescence yield (ph/MeV) for the $337 \mathrm{~nm}$ band at $1013 \mathrm{hPa}$ and $293 \mathrm{~K}$ have been obtained. We will compare below two set of values, i.e., those using the assumptions/calculations of the authors (uncorrected values) and those resulting from our corrections (corrected values). The results presented here are updated values of those previously shown in [13] after some improvement in the simulation code.

\section{Simulation of fluorescence yield experiments}

We have performed a detailed simulation for the experiments of Nagano et al. [2], AirLight [5], FLASH-2008 [6] and MACFLY [4]. Experiments of Kakimoto et al. [1] and Lefeuvre et al. [3] have been also analyzed although a dedicated simulation was not carried out. In table 1 the main parameters of these experiments are displayed.

The Nagano et al. experiment [2] has been simulated including the ge- 
ometry of the collision chamber (see figure 1) as well as other experimental features, e.g., electron-photon coincidence triggered by an electron hitting the scintillator, the light-blocking effect of the collimation channel and the scintillator enclosure. As input to the simulation, we have used a truncated gaussian fit to the measured ${ }^{90} \mathrm{Sr}$ spectrum of [14], with mean energy of $0.85 \mathrm{MeV}$, end point at about $2 \mathrm{MeV}$ and a threshold of $0.3 \mathrm{MeV}$. According to our simulation, most beam electrons of low energy are scattered away at atmospheric pressure and therefore the spectrum of contributing electrons is shifted to higher energies, with a mean value of $1.11 \mathrm{MeV}$ at atmospheric pressure. The statistical uncertainty of this simulation was below $1 \%$.

The approximations made by Nagano et al. are the following. Firstly, they assumed that all the fluorescence is emitted from the beam itself while a fraction of the light is produced by high-energy secondaries well outside

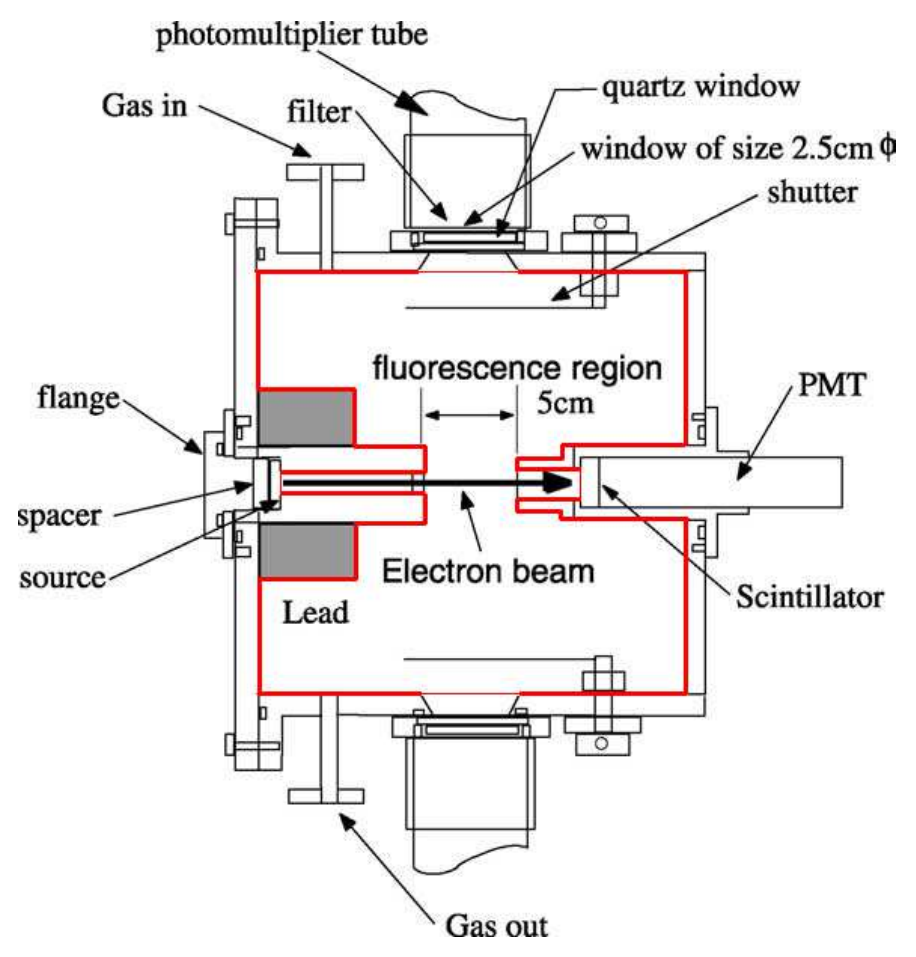

Figure 1: Schematic drawing of Nagano et al. experiment (taken from [14]). Electrons from a ${ }^{90} \mathrm{Sr}$ source are beamed and detected by a scintillator counter. The thick contour represents the geometry implemented in our simulation. 
the beam region. Secondly, they calculated the number of photons per meter assuming they are emitted in a length equal to the gap distance $\Delta x_{\text {gap }}=$ $5 \mathrm{~cm}$, and thus neglecting the dispersion of beam electrons. Finally, for the calculation of the fluorescence yield, they assumed that the energy deposited in the observation volume equals the collisional energy loss $(\mathrm{d} E / \mathrm{d} x)_{\text {loss }}$ at $0.85 \mathrm{MeV}$ as given by the Bethe-Bloch formula. Therefore, the fluorescence yield value $Y_{\mathrm{Nag}}$ reported by Nagano et al., should be corrected by three factors accounting for the above mentioned approximations:

$$
Y=Y_{\mathrm{Nag}} \frac{\Omega_{\text {beam }}}{\Omega} \frac{\Delta x_{\text {gap }}}{\Delta x} \frac{(\mathrm{d} E / \mathrm{d} x)_{\text {loss }}}{\langle\mathrm{d} E / \mathrm{d} x\rangle_{\mathrm{dep}}} .
$$

From our simulation we have found that the acceptance correction, i.e., the $\Omega / \Omega_{\text {beam }}$ increases the fluorescence yield in about $1 \%$. However this effect is nearly compensated by that of the gap length (same size but opposite direction). From our simulation, we have obtained the average energy deposited per electron and unit path length $\langle\mathrm{d} E / \mathrm{d} x\rangle_{\text {dep }}$ as the ratio between the integrated energy deposition and the total path length of beam electrons within the observation volume giving a value somewhat smaller than $(\mathrm{d} E / \mathrm{d} x)_{\text {loss }}$. As a result, according to our simulation, the fluorescence yield of Nagano et al. should be increased by $6 \%$.

The result of the deposited energy from our detailed simulation of the Nagano et al. experiment is fully compatible with the predictions of the generic simulation for a simple geometry, assuming a sphere of radius $R=$ $5 \mathrm{~cm}$ and an electron energy of $1 \mathrm{MeV}$.

The experimental technique used in AirLight [5] is similar to the one of Nagano et al., but with a collisional chamber larger by about a factor of two. In [5] a detailed simulation of the experiment is carried out using GEANT4, including electron scattering by elements of the chamber (e.g., collimator walls, scintillator). Both beam electrons and secondary ones are thoroughly tracked allowing the authors to determine the geometrical acceptance as well as the energy deposited in the observation volume.

For this experiment we have carried out a simulation including propagation of beam electrons and the geometrical details of the set-up. The aim of our simulation was to calculate the integrated energy deposition as a function of the electron energy to be compared with the results found by AirLight. While at low pressure we have obtained results in fair agreement with those reported in [5] (within $5 \%$ at $50 \mathrm{hPa}$ ), at atmospheric pressure our simulation predicts a larger energy deposition. For instance at $800 \mathrm{hPa}$, deviations range 
from about $5 \%$ at low electron energy up to $20 \%$ at $2000 \mathrm{keV}$. The origin of this discrepancy is still unclear. These simulation results have been analyzed [13] taking into account that AirLight reports fluorescence yield values extrapolated at null pressure together with a set of quenching parameters. As a result we propose a correction of $-7 \%$ to the AirLight fluorescence yield.

The FLASH experiment [6] uses an intense beam of $28.5 \mathrm{GeV}$ electrons from a linear accelerator. We have carried out a detailed simulation to evaluate the energy deposited in the chamber as well as the average geometrical acceptance. Statistical uncertainties were reduced to less than $0.3 \%$. In our algorithm, the observation volume has been approximated by a rectangular prism of dimensions $1.60 \mathrm{~cm} \times 1.67 \mathrm{~cm} \times 72 \mathrm{~cm}$. In figure 2, the resulting values of energy deposition are shown as a function of pressure together with those obtained by FLASH using EGS4 (details in [13]). At low pressures $(\sim 300 \mathrm{hPa})$, both results are in very good agreement while at high pressure the discrepancy is of about 5\%. Notice the different pressure dependence of both simulations. This discrepancy could be due to a different treatment of the density correction, which at such a high electron energy is very important. Just to play, we have recalculated the deposited energy with the

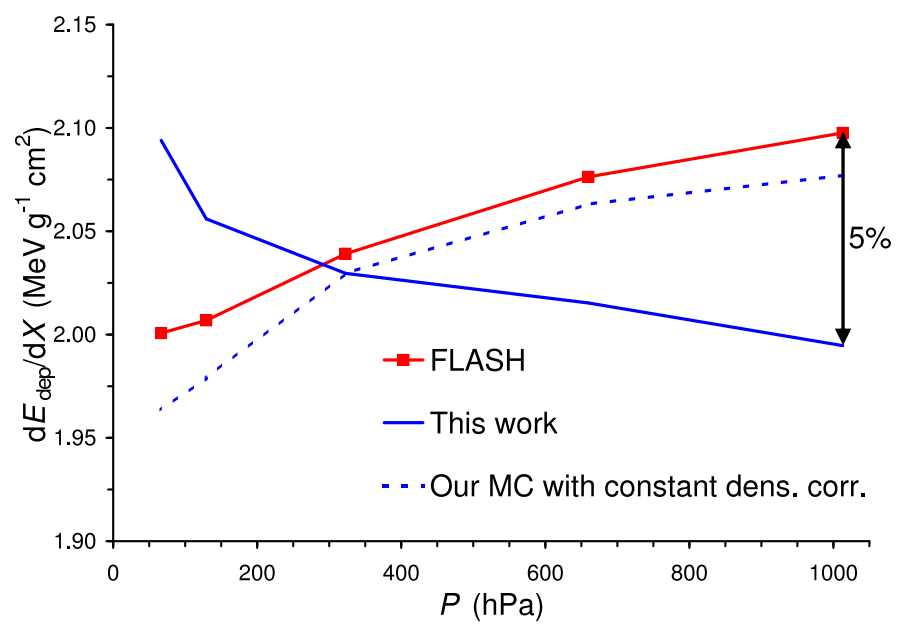

Figure 2: Energy deposited per unit mass thickness as a function of pressure for the FLASH experiment [6]. The largest disagreement between our calculations and the simulations of FLASH is $5 \%$. Note the different behavior of the pressure dependence. For comparison, results from our simulation applying a constant density correction at $323 \mathrm{~h} \mathrm{~Pa}$ in the whole pressure range are also shown. 
Table 1: Comparison of absolute values of fluorescence yield from several experiments. Experimental results as given by the authors are quoted in column 6. Column 9 shows the fluorescence yield in units of photons $/ \mathrm{MeV}$ resulting from the normalization to $337 \mathrm{~nm}, 1013 \mathrm{hPa}$ and $293 \mathrm{~K}$ (dry air) using either the assumptions/calculations of the authors (left) or the results from our simulations (right in bold). See text for details.

\begin{tabular}{|c|c|c|c|c|c|c|c|c|c|}
\hline Experiment & $\begin{array}{c}\Delta \lambda \\
(\mathrm{nm})\end{array}$ & $\begin{array}{c}P \\
(\mathrm{hPa})\end{array}$ & $\begin{array}{c}T \\
(\mathrm{~K})\end{array}$ & $\begin{array}{c}E \\
(\mathrm{MeV})\end{array}$ & $\begin{array}{l}\text { Experimental } \\
\text { result }\end{array}$ & $\begin{array}{c}\text { Error } \\
(\%)\end{array}$ & $I_{337} / I_{\Delta \lambda}$ & $\begin{array}{c}Y_{337} \\
(\mathrm{ph} / \mathrm{MeV})\end{array}$ & $\begin{array}{c}\text { Correction } \\
(\%)\end{array}$ \\
\hline & 337 & 800 & 288 & 1.4 & $5.7 \mathrm{ph} / \mathrm{MeV}$ & 10 & 1 & $4.55 / 4.81$ & 6 \\
\hline Kakimoto [1] & $300-400$ & 1013 & 288 & $\begin{array}{c}1.4 \\
300 \\
650 \\
1000\end{array}$ & $\begin{array}{l}3.3 \mathrm{ph} / \mathrm{m} \\
4.9 \mathrm{ph} / \mathrm{m} \\
4.4 \mathrm{ph} / \mathrm{m} \\
5.0 \mathrm{ph} / \mathrm{m}\end{array}$ & 10 & 0.279 & $\begin{array}{l}4.54 / \mathbf{4 . 8 0} \\
4.44 / \mathbf{5 . 5 3} \\
3.80 / \mathbf{4 . 8 5} \\
4.28 / \mathbf{5 . 5 1}\end{array}$ & $\begin{array}{c}6 \\
25 \\
27 \\
29\end{array}$ \\
\hline Nagano [2] & 337 & 1013 & 293 & 0.85 & $1.021 \mathrm{ph} / \mathrm{m}$ & 13 & 1 & $5.05 / 5.35$ & 6 \\
\hline Lefeuvre [3] & $300-430$ & 1005 & 296 & $\begin{array}{l}1.1 \\
1.5 \\
\end{array}$ & $\begin{array}{l}3.95 \mathrm{ph} / \mathrm{m} \\
4.34 \mathrm{ph} / \mathrm{m}\end{array}$ & 5 & 0.262 & $\begin{array}{l}5.15 / \mathbf{5 . 5 2} \\
5.63 / \mathbf{6 . 1 0}\end{array}$ & $\begin{array}{l}7 \\
8 \\
\end{array}$ \\
\hline MACFLY 4] & $290-440$ & 1013 & 296 & $\begin{array}{c}1.5 \\
20 \cdot 10^{3} \\
50 \cdot 10^{3}\end{array}$ & $\begin{array}{l}17.0 \mathrm{ph} / \mathrm{MeV} \\
17.4 \mathrm{ph} / \mathrm{MeV} \\
18.2 \mathrm{ph} / \mathrm{MeV}\end{array}$ & 13 & 0.255 & $\begin{array}{l}4.32 / \mathbf{4 . 3 5} \\
4.42 / \mathbf{4 . 3 4} \\
4.62 / \mathbf{4 . 5 3}\end{array}$ & $\begin{array}{c}1 \\
-2 \\
-2\end{array}$ \\
\hline FLASH [6] & $300-420$ & 1013 & 304 & $28.5 \cdot 10^{3}$ & $20.8 \mathrm{ph} / \mathrm{MeV}$ & 7.5 & 0.272 & $5.55 / \mathbf{5 . 6 5}$ & 2 \\
\hline AirLight [5] & 337 & - & - & $0.2-2$ & $Y^{0}=384 \mathrm{ph} / \mathrm{MeV}^{a}$ & 16 & 1 & $5.83 / \mathbf{5 . 4 0}$ & -7 \\
\hline $\mathrm{AIRFLY}^{b}[7]$ & 337 & 1013 & 293 & $120 \cdot 10^{3}$ & $5.6 \mathrm{ph} / \mathrm{MeV}$ & $\lesssim 5 \%$ & 1 & $5.6 /-$ & - \\
\hline
\end{tabular}

density correction for $323 \mathrm{hPa}$ in the whole pressure range. Now the resulting energy dependence follows a similar behavior (see figure 21).

The results of our detailed simulation for the FLASH experiment are in excellent agreement (within 1\%) with that obtained from the generic simulation for a sphere of $1.67 \mathrm{~cm}$ radius.

The absolute calibration of the optical system of FLASH was carried out by comparison with the measurement of Rayleigh-scattered light from a narrow beam of a nitrogen laser. They found that efficiency of the optical system for fluorescence detection is $(3.2 \pm 0.25) \%$ lower than that for the calibration laser beam, due to the wide-spread energy deposition from the electron beam. However, our simulation predicts that this effect is negligible in the whole pressure range for this set-up. Therefore according to our simulation the fluorescence yield reported by FLASH should be increased by $2 \%$.

The MACFLY experiment [4] performed measurements at 20 and $50 \mathrm{GeV}$ using electrons from a linear accelerator and $1.5 \mathrm{MeV}$ (mean energy) from 
a ${ }^{90} \mathrm{Sr}$ source. The energy deposited in the field of view is calculated by the authors with GEANT4. We have also carried out a detailed simulation with the following features. Primaries colliding the walls of the chamber or being stopped inside, and so not reaching the trigger system, are rejected in our simulation. The average energy deposited per electron and unit mass thickness has been obtained from the ratio between the integrated energy deposition and the total path length of triggering primaries. Statistical uncertainties were reduced below $0.5 \%$. The deposited energy obtained from our simulation is very close to that reported by MACFLY. According to our simulations the fluorescence yield of MACFLY should be increased by $1 \%$ at $1.5 \mathrm{MeV}$ and decreased by $2 \%$ at 20 and $50 \mathrm{GeV}$. Again the detailed simulation is in good agreement (in this case better than $2 \%$ ) with the generic simulation for $R=10 \mathrm{~cm}$ at energies above $0.5 \mathrm{MeV}$.

For the experiments of Kakimoto et al. [1] and Lefeuvre et al. [3] we have compared the predictions of our generic simulation with those assumed by these authors. In the Kakimoto et al. experiment electrons with a mean energy of $1.4 \mathrm{MeV}$ from a ${ }^{90} \mathrm{Sr}$ source as well as those from an electron synchrotron with energies of 300, 650 and $1000 \mathrm{MeV}$ were used. Fluorescence light was produced and observed in a $10 \mathrm{~cm}$ gap. For the determination of the fluorescence yield, they assumed full energy deposition in the beam axis. According to our generic simulation, the deposited energy inside a sphere of $10 \mathrm{~cm}$ radius is about $6 \%, 25 \%, 27 \%$ and $29 \%$ lower than the energy loss for 1.4, 300, 650 and $1000 \mathrm{MeV}$ electrons, respectively. Therefore, the fluorescence yield values at the above energies should be increased correspondingly by these factors. Results obtained from applying these corrections to measurements of Kakimoto et al. are consistent with those obtained in [16] using an alternative procedure.

In the experiment of Lefeuvre et al. [3], electrons with mean energies of $1.1 \mathrm{MeV}$ (or $1.5 \mathrm{MeV}$ when a higher energy threshold is applied to the electron detector) from a very intense ${ }^{90} \mathrm{Sr}$ source $(370 \mathrm{MBq})$ were used.

From our generic simulation, assuming a sphere of $4 \mathrm{~cm}$ radius for Lefeuvre et al. experiment, we found that the deposited energy is $9 \%$ and $10 \%$ lower than the total energy loss at mean electron energies of 1.1 and $1.5 \mathrm{MeV}$, respectively. However, electron scattering by the lead shield of this set-up is not included in our simulation, which assumes that secondaries reaching the walls are absorbed, and thus, it may underestimate the deposited energy. Taking into account this effect (see [13] for details), the fluorescence yield of [3] should be increased by $7 \%(8 \%)$ for $1.1(1.5 \mathrm{MeV})$. 

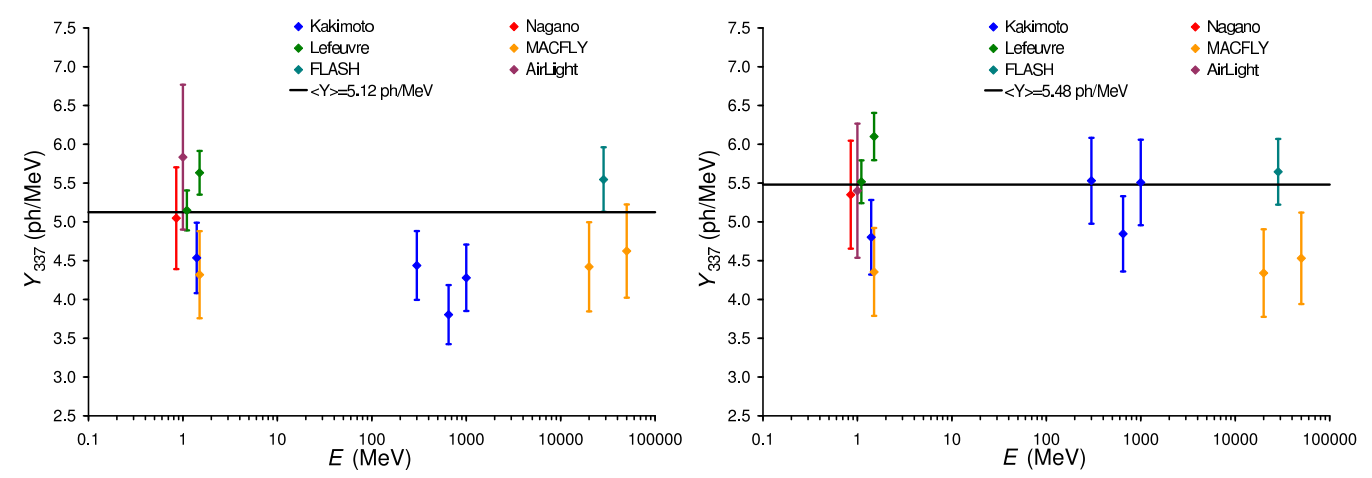

Figure 3: Comparison of normalized $Y_{337}$ values as a function of energy for both uncorrected (left) and corrected (right) values. The horizontal lines represent the corresponding weighted average values.

\section{Comparison of fluorescence yield values [15]}

The air-fluorescence yield measurements are listed in table 1 indicating the experimental conditions (i.e., pressure, temperature, energy, wavelength, units) as well as the uncertainties (column 7). The normalization factor to get the fluorescence yield for the $337 \mathrm{~nm}$ band $I_{337} / I_{\Delta \lambda}$ is shown in column 8 . The value of $Y_{337}$ defined as the fluorescence yield at $1013 \mathrm{hPa}$ and $293 \mathrm{~K}$ in $\mathrm{ph} / \mathrm{MeV}$ units is shown in column 9. Values inferred from the assumptions (or calculations) on energy deposition and geometrical factors given by the authors are displayed in left side. In bold the results obtained after applying the corrections proposed in the previous section (column 10) are shown (column 9 - right).

Several conclusions can be extracted from this table. The uncorrected normalized values are in reasonable agreement although discrepancies are very often beyond experimental uncertainties. On the other hand, the proposed corrections are non-negligible $(6 \%$ - 29\%) for experiments where the energy deposition is approximated by the electron energy lost, i.e., [1, 2, 3]. In general, our simulations are in good agreement with those carried out by the experiments $(\lesssim 2 \%$ for MACFLY and FLASH). In regard to AirLight we have found some discrepancy (proposed correction of $7 \%$ ) but its origin is still under discussion.

Normalized fluorescence yields have been represented as a function of the electron energy in figure 3, both uncorrected (left panel) and corrected (right panel) values. As can be appreciated, measurements are in better agreement 

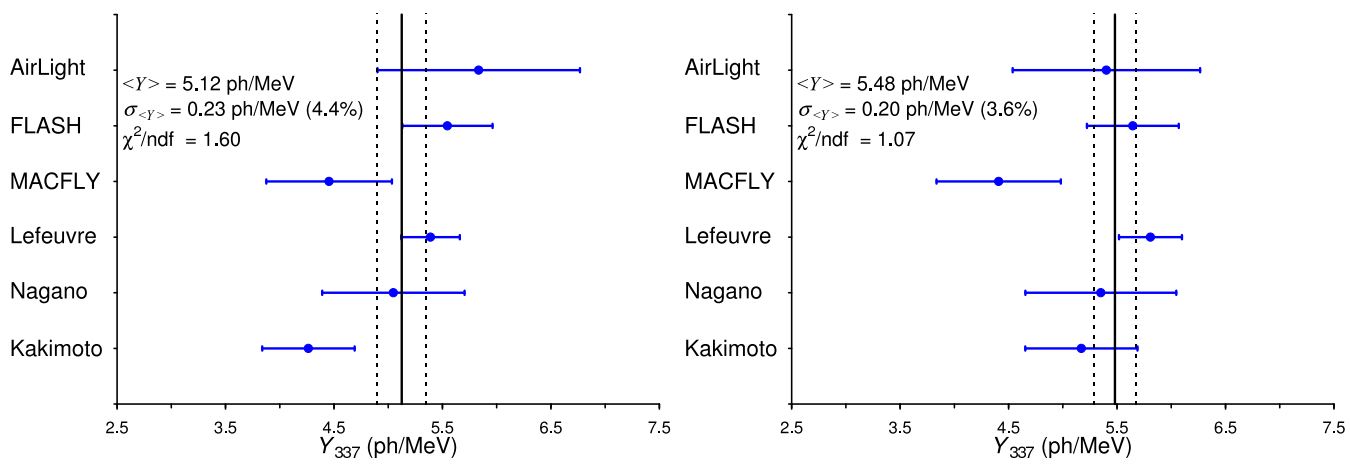

Figure 4: Graphical representation of the $Y_{337}$ values at $1013 \mathrm{hPa}$ and $293 \mathrm{~K}$ for both uncorrected (left) and corrected (right) values. The weighted mean $\langle Y\rangle$ (vertical continuous line), the standard deviation of the mean $\sigma_{\langle Y\rangle}$ (half of interval between dashed lines) and the $\chi^{2}$ statistic normalized by the number of degrees of freedom are shown in the legends. Better agreement and consistency of the data sample are found in the right plot.

when including our corrections. In addition, the corrected results give more support to the expected energy independence of the fluorescence yield.

In order to know quantitatively to what extent our corrections favor the agreement between the absolute results included in this comparison, a statistical analysis has been performed. In the first place, for a given experiment, results obtained at different energies have been averaged assuming a common systematic error. Then, the average value of this sample $\langle Y\rangle$ has been calculated weighting the data with the reciprocal of the quoted square uncertainties shown in column 7 of table1(i.e., $w_{i}=1 / \sigma_{i}^{2}$ ). Also the corresponding variance $\left(\sum_{i} 1 / \sigma_{i}^{2}\right)^{-1}$ and the $\chi^{2}$ statistic divided by the number of degrees of freedom has been computed. The $\chi^{2} /$ ndf result is larger than 1 for the uncorrected sample, indicating that the quoted uncertainties are very likely underestimated due to the fact that authors usually do not include any error contribution from the evaluation of the deposited energy. For the calculation of the uncertainty in the average fluorescence yield $\sigma_{\langle Y\rangle}$, the variance has been multiplied by $\chi^{2} /$ ndf, following the usual procedure.

The results shown in figure 4 for both uncorrected (left panel) and corrected (right panel) values indicate that our corrections lead to a more consistent data sample suggesting that they do improve the determination of the deposited energy for the different experiments.

We have checked [15] that the weighting procedure has no significant 
effect on the final result. In addition, removing each value while keeping the remaining ones in the corrected sample does not significantly change the weighted average. The corresponding results range from $5.61 \mathrm{ph} / \mathrm{MeV}$ (removing MACFLY) to $5.23 \mathrm{ph} / \mathrm{MeV}$ (removing Lefeuvre et al.). A detailed analysis of these features [15] including the effect of a weak energy dependence of the fluorescence yield in the $0.1-10 \mathrm{MeV}$ range, predicted by our simulations, led us to an average value of $Y_{337}=5.45 \mathrm{ph} / \mathrm{MeV}$ with a conservative estimated error of $5 \%$. According to the comparison of our simulation result on energy deposition with GEANT4, a small systematic uncertainty of below $2 \%$ should be added, although it does not affect the $\chi^{2}$ value of the corrected sample.

The recent absolute measurement of the AIRFLY collaboration [7] yields $Y_{337}=5.6 \mathrm{ph} / \mathrm{MeV}$ with an uncertainty of $\lesssim 5 \%$ (still preliminary), which is fully compatible with the above value. If this new result is included in the average, then a weighted mean value of $5.52 \mathrm{ph} / \mathrm{MeV}$ is obtained with an uncertainty of $\lesssim 5 \%$.

As already mentioned, for the comparison presented here we have normalized the air-fluorescence yield measurements to its value for the $337 \mathrm{~nm}$ band at $1013 \mathrm{hPa}$ and $293 \mathrm{~K}$. However, in some occasions it might be more convenient to use the integral of the fluorescence yield in a wider spectral range and/or other pressure and temperature conditions. The conversion can be easily done following the procedure described in detail in [10]. For instance, the above average value would be of $20.1 \mathrm{ph} / \mathrm{MeV}( \pm 5 \%)$ for the $300-420 \mathrm{~nm}$ spectral range at the same reference pressure and temperature, which would become $20.3 \mathrm{ph} / \mathrm{MeV}$ if the measurement of AIRFLY is included.

Our simulation can also provide a theoretical value of the air-fluorescence yield. Unfortunately, the evaluation of the fluorescence emission cannot be very precise due to the large uncertainties in the relevant molecular parameters. Therefore, we expect a large uncertainty in such a calculation of the fluorescence yield, which we estimated to be about 25\% [10]. Nevertheless, a result of $Y_{337}=6.3 \mathrm{ph} / \mathrm{MeV}$ (using the quenching parameter of [11]) has been found, which is consistent with the experimental ones, providing a valuable theoretical support to these measurements. 


\section{Conclusions}

Available measurements of the absolute air-fluorescence yield have been normalized to common conditions (1013 hPa, $293 \mathrm{~K}, 337 \mathrm{~nm})$ and units (ph/MeV). According to the simulations presented in this work, experimental results obtained neglecting the energy deposited by secondary electrons outside the field of view of the optical system have to be corrected by non-negligible factors ranging from 6 to $29 \%$. Our evaluation of energy deposition is, in general, in agreement with that reported by those experiments which carried out a detailed simulation. The corrections to the measurements of the absolute air-fluorescence yield proposed here increase significantly the compatibility of experimental results. An average value of $Y_{337}=5.45 \mathrm{ph} / \mathrm{MeV}$ with a $5 \%$ uncertainty has been obtained. If the absolute fluorescence yield and error of AIRFLY are confirmed, a consensus on this important parameter with an uncertainty below the $5 \%$ level could be reached with high reliability.

\section{Acknowledgements}

This work has been supported by the Spanish Ministerio de Ciencia e Innovacion (FPA2009-07772 and CONSOLIDER CPAN CSD2007-42) and "Comunidad de Madrid" (Ref.: 910600). J. Rosado acknowledges a PhD grant from "Universidad Complutense de Madrid". The authors thank our colleagues of the Auger collaboration for fruitful discussions and comments on this work.

\section{References}

[1] F. Kakimoto et al., Nucl. Instr. and Meth. A 372527 (1996).

[2] M. Nagano et al., Astropart. Phys. 22235 (2004).

[3] G. Lefeuvre et al., Nucl. Instr. and Meth. A 57878 (2007).

[4] P. Colin et al. [MACFLY Collaboration], Astropart. Phys. 27317 (2007).

[5] T. Waldenmaier et al., Astropart. Phys. 29205 (2008).

[6] R. Abbasi et al. [FLASH Collaboration], Astropart. Phys. 2977 (2008).

[7] P. Privitera, Proceedings of this conference. 
[8] F. Blanco and F. Arqueros, Phys. Lett A 345355 (2005).

[9] F. Arqueros et al., Astropart. Phys. 26231 (2006).

[10] F. Arqueros et al., New J. Phys. 11065011 (2009).

[11] M. Ave et al. [AIRFLY Collaboration], Astropart. Phys. 2841 (2007).

[12] The AIRFLY Collaboration, private communication.

[13] J. Rosado et al., Astropart. Phys. 34164 (2010).

[14] M. Nagano et al., Astropart. Phys. 20293 (2003).

[15] In this section we show preliminary results of a work in preparation (available in arXiv:1103.2022)

[16] F. Arqueros et al., Proceedings of 43rd Rencontres de Moriond - Electroweak Interactions and Unified Theories (La Thuile, Italy, 2008). Available in arXiv:0807.4824 